\title{
The Next Pandemic: Supporting COVID-19 Frontline Doctors Through Film Discussion
}

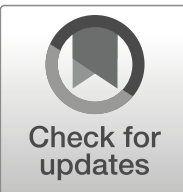

\author{
Cristelle Chow $^{1} \cdot$ Raveen Shahdadpuri $^{1} \cdot$ Kam Kai-Qian $^{1} \cdot$ Chan Yoke Hwee $^{2}$ \\ Published online: 5 September 2020 \\ (C) Springer Science+Business Media, LLC, part of Springer Nature 2020
}

\begin{abstract}
This paper describes an innovative just-in-time health humanities programme to educate and provide support to COVID-19 frontline doctors-in-training. The programme incorporates small-group screening of the Netflix documentary, The Next Pandemic from the Explained series, followed by a one-hour facilitated discussion to explore themes surrounding the current pandemic and its impact on frontline doctors in a tertiary paediatric hospital in Singapore. Themes derived from the film included preparedness, blame, and the impact on healthcare workers and public, which were further discussed to include concerns regarding current local readiness levels given global connectivity, the need for international cooperation, and the effects of blame such as racism and prejudice. The association with culture; the current impact on healthcare workers, physician-patient relationships, and the public including the role of social media, the government and associated public reactions were also explored. These rich discussions demonstrate the pivotal role health humanities has in times of uncertainty such as an emerging infectious disease outbreak by providing timely pandemic education and supporting reflective learning.
\end{abstract}

Keywords Film $\cdot$ Pandemic $\cdot$ Reflective learning $\cdot$ Postgraduate education

\section{Introduction}

The term, "Cinemeducation," coined by Alexander, Hall, and Pettice $(1994,430)$, refers to the use of movie clips to educate medical students and residents about the psychosocial aspects of medicine. Films, because of their audio-visual nature, can both engage and enthral viewers with emotional and dramatic portrayals of individual struggles and challenging interpersonal relationships. When health conditions, whether physical or psychological, drive or are

Cristelle Chow

cristelle.chow.ct@singhealth.com.sg

1 Department of Paediatrics, KK Women's and Children's Hospital, Singapore, Singapore

2 Department of Paediatric Subspecialties, KK Women's and Children's Hospital, Singapore, Singapore 
explored in the film's themes, viewers can gain a deeper appreciation of the illness experience (Powley and Higson 2005). Especially for individuals training in the healthcare professions, movies can engage learners in deeper discussion and promote active learning through cognitive and social constructivism. Learners embed new knowledge acquired from the film onto pre-existing cognitive structures and construct knowledge through group discussions with other learners, reflecting upon their personal experiences along the way. A systematic review by Darbyshire and Baker (2012) provides insights into the various areas in which cinema is used in medical education, ranging from hard science topics like biology and virology to soft skills like professionalism and ethics.

While movies can be used for the teaching of many aspects of medicine, the use of movies in providing education and support during acute outbreak situations has yet to be described, as the health humanities is often viewed as a component of "peace time" education. The uncertainty of an emerging infectious disease can have significant psychological impact on frontline doctors (Tan 2020; Lai et al. 2019), and provision of the health humanities during this period could potentially help to provide just-in-time education and address perceptions of safety, threat and risk (Rambaldini et al. 2005).

Hence, as the world experiences the current COVID-19 pandemic situation, this study aims to describe the use of a short film and post-film discussion to educate and support frontline doctorsin-training during an acute emerging infectious disease outbreak. This study also aims to broadly describe the discussion themes generated through this just-in-time intervention (Table 1).

\section{Methods}

\section{Participants, facilitators and setting}

From 10 February 2020 onwards, the Department of Paediatrics in a tertiary women's and children's hospital in Singapore assigned rotating groups of house medical staff and paediatric consultants into isolation wards designated for suspect and confirmed COVID-19 paediatric cases. Rotations for house medical staff were in two-week blocks including weekends and public holidays, in a twelve-hour shift system. Dyads consisting of related paediatric and adult patients were also admitted to these wards. In mid-February 2020, Singapore experienced an

Table 1 Discussion Themes

Main Themes from Film

Preparedness

Allocation of Blame

Impact on Healthcare Workers

Impact on the Public

\section{Branched Themes During Film Discussion}

Current preparedness levels

Global connectivity

Need for international cooperation

Racism and prejudice

Influence of culture

Perception of safety

Fears of healthcare workers and families

Discrimination against healthcare workers

Physician-patient relationships

Social media

Reactions of the public

Role of the government

Appreciation for healthcare workers 
increasing number of COVID-19 infected cases in the adult healthcare institutions. In our institution, we had admitted a dyad of infected infant and mother in our isolation unit by 10 February 2020 (Kam et al. 2020). There was still significant uncertainty about the virus' transmissibility, virulence and potential to cause morbidity and mortality. All house medical staff posted to isolation wards, consisting of freshly graduated house officers, medical officers (in non-training positions), paediatric junior residents, senior residents and resident physicians were invited to the film screening during their shift hours. Participation was voluntary, and all participants were informed that a post-film discussion with notetaking by the facilitators would be carried out. Facilitators consisted of paediatric faculty from general paediatrics, intensive care and infectious diseases, and some facilitators were teleconferenced into the discussion due to the hospital's enforced modular system.

\section{Film selection and preparation}

The film was selected based on recommendations by Baños and Bosch (2015). Two faculty members (CC and RS) reviewed several pandemic-related films of varying durations to assess its suitability and relevance for this study. The decision was made to screen the short documentary from the original Netflix Explained series entitled, The Next Pandemic. It was selected due to its timely recent release (7th November 2019), directness, factuality, short runtime of just over twenty minutes, relevance to the current hospital climate, and feature of Severe Acute Respiratory Syndrome (SARS) which significantly impacted Singapore in 2003 (Tan 2006). The above-mentioned faculty members independently watched the film multiple times to determine its underlying themes, generate educational objectives and questions to be used in the post-film discussion. The educational objectives of the session were: (1) To know the significant historical pandemics that impact ongoing pandemic preparedness efforts, (2) To discuss the challenges associated with the current pandemic and (3) To reflect on the impact of the current pandemic on personal lives, healthcare workers and the general public. Three questions were selected for post-film discussion: (1) How did the film make you feel about the current COVID-19 situation? (2) How has frontline COVID-19 work impacted your personal lives, your families and your relationships with your patients? (3) How do you feel about the public's response to the COVID-19 pandemic?

\section{Session description}

The session started with projection of the documentary to a small group of not more than twelve participants. This was followed by a post-film discussion facilitated by faculty members for approximately one hour. Facilitators encouraged active participation using the prepared questions although new and more relevant issues emerged during the session. Participants and faculty members interactively exchanged their opinions in a nonjudgemental and constructive way, using the film as the initial basis of discussion, then branching out into new discussion areas. During the session, facilitators took notes of the broad themes discussed, including relevant quotes from participants.

\section{Data analysis}

Notes taken by facilitators were thematically analysed by the study authors independently for recurring themes. This was followed by discussion among the authors, and the final themes 
that were mutually agreed were organized in relation to the pre-determined themes from the film.

\section{Results}

A total of six similar sessions were conducted between 10 February and 6 March 2020, involving a total of sixty-three participants and six faculty members. Of the sixty-three participants, twenty-eight were paediatric senior residents and resident physicians, four were paediatric junior residents, fourteen were non-trainee medical officers, and fifteen were house officers. Of the faculty members, three were paediatric infectious disease physicians, two were general paediatricians, and one was a paediatric intensivist.

From the film, four issues in particular were depicted as the main themes for group discussion, which included the following: pandemic preparedness, the allocation of blame, the impact on healthcare workers and the impact on the general public. During the film discussion, these were further branched into other areas of discussion by the participants (Table 1).

\section{Preparedness}

\section{Current preparedness levels}

Participants were asked the question of whether the country, hospital and the public were truly prepared for the pandemic. They reflected upon the film's depiction of SARS, and how the authorities in affected countries had "let it pass" until there was a significant number of deaths. They questioned whether we had really learnt from the SARS outbreak and expressed helplessness, fear, uncertainty and being overwhelmed by the possibility of an uncontrollable pandemic. These perceptions were more prevalent in the discussions that were held in midFebruary 2020 when the outbreak had still not peaked. A few residents shared that their fear stemmed from the uncertainty of how lethal the virus was and that preparation, vaccine research, personal protective equipment and border controls may not be sufficient to control the spread of the virus should it be virulent and highly transmissible. In discussions held in late-February and early March, participants expressed less concern about preparedness despite the increasing number of suspect and confirmed cases, as no healthcare workers were infected, and mortality rates were lower than SARS. Some of them also mentioned their personal preparedness and comfort due to the presence of adequate personal protective equipment and training: "I don't think anyone is really fearful to be in the EID team - that says a lot about our preparedness."

\section{Global connectivity}

Many comparisons were made between SARS and COVID-19 with one of the largest differences being the current increased global connectivity of humans because of the affordability and availability of air travel. As Singapore is an international transport, trade and travel hub, participants raised concerns about border control methods and whether they were timely or effective in controlling the spread of the virus to the local population. 


\section{Need for international cooperation}

Participants recognized the need for international cooperation as one resident shared that the film "makes us feel vulnerable... that we should unite as a human race as this virus transcends gender, race, socioeconomic status...." There were genuine concerns raised that the pandemic preparedness was "not high on the agenda of some governments" and that insufficient funding and resources were being designated for this purpose. Participants lamented about the "rise of ultra-nationalism" and self-preservation that would only backfire, as international cooperation and putting aside political and social differences would be key to tackling this pandemic.

\section{Allocation of blame}

\section{Racism and prejudice}

Just as the film subtly apportions blame of the SARS epidemic to China (and its wet markets selling live and wild animals), some residents felt that since the start of the COVID-19 outbreak, this blame was manifesting in some countries as racism and prejudice. Several participants reflected upon the experiences of loved ones in Western countries who were "looked at in a particular way" or treated differently due to being of Chinese descent or Asian ethnicity.

\section{Influence of culture}

The discussion with regards to the role of traditional wet markets was particularly lively as participants shared that Southeast Asian countries still have a thriving live wet market scene, which is still very much the cultural norm. It was acknowledged that while live wet markets where "the meat is fresh" are indispensable to many Asian communities, the consumption of undomesticated wild animals was not widespread. In China, this practice has been typically associated with higher social status and deep-seated beliefs about the perceived nutrition value of exotic meats. Hence, while the general public may be quick to blame others for being the source of the current pandemic, participants reflected that we should also look to our own practices and not be too quick to judge the culture and beliefs of others.

\section{Impact on healthcare workers}

\section{Perception of safety}

While working on the ground, most participants felt safe wearing personal protective equipment (PPE) provided by the hospital. Some even mentioned that they did not flinch when they were coughed upon by patients while wearing PPE, and one participant shared: "We're mask fitted, PAPR (powered air-purifying respirator) trained, and just this feeling that we are well taken care of." However, given initial reports about asymptomatic COVID-19 carriers, some participants were concerned whether they were safe in public areas such as on public transport, as they perceived that they were "more likely to get COVID in (trains) rather than from the ward because of the precautions that we take in hospital." 


\section{Fears of healthcare workers and their families}

Participants had differing opinions on disclosure to their families about their hospital work. Some participants, especially those who had family members who were also healthcare workers $(\mathrm{HCW})$, were open about their role in the isolation ward. Some of them declared to their loved ones, "I'm dirty... I'm a potential centre for a new cluster" and voluntarily cancelled their social appointments for the period when they worked in the isolation wards. Others had decided to "censor information," as they did not want their family members to fear for their well-being. One participant shared that it was "tiring reasoning with every patient then going home and reasoning with family."

Most family members were supportive of the participants' jobs especially if they were kept informed about the hospital environment and measures taken to minimise transmission to healthcare workers. Family members who were also healthcare personnel tended to express more understanding and less paranoia around the fact that the participants were looking after potentially infected or infected patients. However, some participants shared that their family members were still anxious and fearful of contracting the virus from the participants. One frontline staff mentioned that his parents called him "a virus" and kept their distance from him whilst at home, while others provided anecdotes about family members refusing to meet them at social events especially if they were elderly or parents of young children. However, the participants respected the wishes of their loved ones, as one shared, "I can't blame them, they are not wrong, (the elderly person) is more likely to develop a more severe illness."

\section{Discrimination against healthcare workers}

While most participants did not personally experience any overt HCW discrimination, a few shared thoughts about news circulating on social media that made them concerned about this rising trend. It was reported that local nurses in hospital uniforms were chased off public transport and told to leave public eateries due to public fears about contracting the virus from $\mathrm{HCW}$. One participant recounted an episode of a private hire car driver cancelling his ride to the hospital when the participant shared that he was a doctor going to work. In later sessions, participants noted that there were fewer reports of discrimination against HCW and that the public had started to show more support through notes of encouragement, as well as retail, food and beverage promotions and discounts from local businesses.

\section{Physician-patient relationships}

Communication with caregivers was deemed the most important task whilst working in the isolation ward. Although it was time-consuming, participants felt that caregiver reassurance was key to ensuring adherence to hospital isolation policies and recommended treatment plans. One resident reflected the importance of "having a bit more of a personal touch... reassuring (caregivers) that measures are being taken and the reasons why isolation is as such." Anxiety levels of caregivers in the isolation ward were felt to be higher compared to the other general wards, not only due to COVID-19 concerns but also due to the heightened movement restrictions associated with isolation ward admissions. With the need for constant PPE use, there was more physical distance between physicians and patients, inability to see the HCW facial expressions and other non-verbal cues, some challenges in obtaining language interpreters, breathlessness with N95 masks and fogging of goggles resulting in physicians' 
discomfort and hence shorter-than-desired consultations. There were also discernible fears of some young children in seeing healthcare staff wearing "spacesuits" especially when obtaining nasopharyngeal specimens for viral testing, although participants tried to reduce patient anxiety through "waving from outside and walking in slowly."

\section{Appreciation for colleagues/ other healthcare workers}

Participants mostly felt that stepping up to the frontline during a disease outbreak was their duty and one of the reasons for entering the medical profession. In response to individuals who had reservations about working in isolation wards, one participant reflected, "We entered this profession for a reason... in a time of need, how can others refuse to do their duty?" There was heightened appreciation for non-medical healthcare workers, including nurses, who must "stay in the ward and wear the N95 throughout their shift," porters, lab technicians and ancillary staff such as the porters and cleaners. One participant described watching cleaners wearing N95 masks who were diligently detaching and disinfecting wheelchair components and was "very impressed by the effort."

\section{Impact on the public}

\section{Social media}

A significant difference between the time of SARS and the current COVID-19 pandemic is the widespread ubiquity and availability of social media (e.g. Whatsapp, Facebook, Instagram). Social media was viewed as a "double-edged sword"; on one hand, it provides the public with government-sanctioned information and updates, but on the other hand, it can spread fake news and fuels unwarranted rumours and panic. Through social media, personal stories can surface, and different sides of the story can be published - for example, one participant shared the heart-warming posts of Chinese citizens caring for each other during the Wuhan lockdown, which contrasted with undercover videos of Chinese hospitals dealing with throngs of patients. Overall, social media was still viewed as a positive tool for information dissemination, as long as it was adequately regulated and the public was directed to appropriate and reliable sources of information.

\section{Reactions of the public}

Participants were collectively concerned about the "uninformed public," as reflected through panic buying and hoarding of daily necessities when the Ministry of Health escalated to a higher level of Disease Outbreak Response System Condition (also known as DORSCON) from Yellow to Orange on 7th February 2020. Controlling public reaction was viewed as a major challenge as participants felt that they could not influence members of the public who refused to abide to government recommendations - "If people do not buy in, (we) cannot force it down." However, participants recognized that as healthcare workers, it was also their responsibility to provide accurate information to the public, whether to concerned family members or doubting caregivers in order to increase public trust in government authorities and reduce irrational and selfish public behaviour. It was also agreed that healthcare workers should be consistent in their messaging and set good examples in following government advisories such as avoiding panic buying and hoarding behaviour and not wearing N95 masks 
in public places where it is not clinically necessary (as this would lead to over consumption of limited N95 supplies). One resident shared that healthcare workers were key to "educating the public to really filter through information that is coming through... and how to react to things when they happen."

\section{Role of the government}

Most participants felt that the government had built up public trust through decisive responses and transparent decision-making processes. They felt that they could trust the authorities to take the necessary steps, whether in border control measures, healthcare advisories or contact tracing efforts in order to protect the local population and reduce the virus' transmission within the community. However, there was some debate on whether the extent of disclosure of positive cases' personal information to the public domain was appropriate, as there was concern about the stigma experienced by positive cases and their family members. One resident shared a personal experience of a close family friend who had recently returned from Wuhan, the first epicentre of the virus, and the discrimination that the family experienced despite being tested negative for COVID-19. Participants recognized the fine balance between government transparency and personal data protection, and that "there must be bodies in place to scale down and to decide on what is necessary and what is not," as this would also change as the pandemic evolves.

\section{Appreciation for healthcare workers}

Although a handful of participants were initially sceptical about the public's demonstration of appreciation for the HCWs, gestures including hand-written notes of gratitude, donation of food items and artworks were generally welcome. Some participants also shared that caregivers of both positive and suspect patients had verbally expressed their appreciation to the healthcare team during ward rounds, and admitted children had drawn thank-you cards for nurses and doctors. These simple gestures served as positive motivators for the frontline team.

\section{Discussion}

Our study demonstrates the feasibility of a short documentary film in generating reflective discussions about an ongoing pandemic and its impact on frontline workers. This health humanities intervention primarily uses the principles of reflection and experiential learning in medical education. Reflection, the metacognitive process that creates greater understanding of self and situations to inform future action, has been used in various medical educational approaches (Sandars 2009). In the health humanities, reflection is encapsulated in the studentcentred affective developmental approach, which focuses on the development of self-concept, insight and introspection through the exploration of feelings, attitudes and values (Self 1993). Guided reflection through discussions, rather than reflective writing, was used in this intervention in order to provide participants a safe and supportive environment to make sense of their personal experiences, through non-judgmental questioning and acceptance of different opinions within the group (Sandars 2009). Incorporating the principles of the experiential learning cycle, reflection is the second phase after experience, followed by "abstract conceptualisation" (Kolb 2014, 50). In this manner, as participants watch the film, they reflect upon 
how the themes presented through the film relate to their personal experiences of working in the isolation wards during the COVID-19 pandemic. As they share these reflections during the film discussion, they conceptualize new ideas about the ongoing pandemic including its impact on their personal lives, the healthcare system, government authorities and society. Being a collaborative group discussion, aspects of social constructivism also contribute to the learning process, as the participants' knowledge is co-constructed through learning from each other's personal experiences and opinions (Vygotsky 1978). It is also hoped that this intervention provides peer support and encouragement to the participants, improving their mental wellness as they work in the high-risk areas during this pandemic.

Some of the themes that emerged from the discussion were similar to those reported in other studies that explored the impact of emerging infectious diseases on house staff or medical residents. For example, during the SARS outbreak in Toronto, medical residents expressed concerns about personal safety and the wellbeing of loved ones, their sense of duty to care, as well as feelings about social isolation (Rambaldini et al. 2005). While study participants felt confident about PPE and hospital infection control policies, they were also worried about asymptomatically passing on the virus to their loved ones as asymptomatic transmission of SARS-CoV-2 was a real possibility, in contrast to the SARS virus. The impact of social isolation, whether self-imposed or imposed by others, could have a minimized impact on study participants compared to frontline workers during SARS, due to the current widespread use of video chats and social media, which were not available in the early 2000s. During the Middle Eastern Respiratory Syndrome (MERS) outbreak, medical residents reported concerns about personal and hospital preparedness and fear of disclosure about their frontline work to family members (Aldrees et al. 2017). Although study participants did share that the film questioned the world's preparedness for the next pandemic, when it came to hospital infection control policies, personal protection and PPE training, the majority of study participants felt adequately prepared about working in isolation wards, and there was significant trust in the government's and hospital's management of the worsening outbreak. Like medical residents in Saudi Arabia, some study participants chose to withhold information about their frontline work to family members. As a conservative Asian society, it is still common practice to withhold information about personal health and safety risks from the affected patient, so as to not cause unnecessary worry and anxiety among family members, especially those who may be elderly or less educated.

The responses of the participants evolved over the course of four weeks with initial fears and anxiety being replaced with more certainty about preparedness. This evolution was likely related to better understanding of the novel coronavirus, the local and global response to the virus, and the extent of control. Participants also experienced the "normalization" of working in an outbreak setting - as HCWs gain acceptance of the crisis and with increasing positive cases, they would experience less apprehension as positive cases start to become the "norm." Towards the last few sessions, it appeared that participants accepted that caring for suspect and positive cases would become the mainstay of their clinical work. However, without a heightened sense of vigilance, this "normalization" may result in a lapse in infection control measures. Hence, it would be prudent to ensure that frontline workers are constantly provided with appropriate infection control and policy updates to ensure that vigilance and selfprotection are still maintained as the pandemic ensues.

An additional pandemic-related theme that has not been previously reported is the apportioning of blame and its subsequent effects of racism and prejudice. In the film, the vivid and somewhat unsettling scenes of Chinese wet markets selling live animals during the SARS 
epidemic could have triggered unhappiness over the similarities to which the source of the SARS and COVID-19 outbreaks may have originated from. While there was frustration about how governments did not seem to learn from past mistakes, participants also had the opportunity to reflect upon the challenges that governments face in banning practices that are deeply rooted in the society and culture. The discussion enabled participants to also reflect on our own personal biases and not let them cloud our judgement of others and their actions.

The doctor's role in the education of the public was also repeatedly discussed. Participants saw themselves as representatives beyond the hospital setting as role models for the public to follow government advisories and to protect patient confidentiality. Their ability to accept this additional responsibility likely stemmed from their reflection on current public behaviours and their considerable impact. This provided them with an opportunity to generate insight on how they perceived their role as healthcare professionals and allowed them to explore the rationale behind their behaviours and attitudes during this time of international crisis (Ginsburg and Lingard 2006). While participants' responses on the transparency and actions of the government may be quite unique to this study's setting, exploration of this theme in other settings would also be a worthwhile endeavour in hospital pandemic responses, as trust in health and government authorities can have a significant impact on the mental wellbeing of healthcare staff.

Finally, it was heartening to see that participants were able to reflect upon the importance of appreciating all staff working in healthcare, and while the public may view the true "heroes" as being doctors and nurses, we need to be mindful of the other silent healthcare workers in our midst who are indispensable in combating this pandemic.

This study is limited by the number of participants, but despite the small sample size, there was significant heterogeneity in the background and experiences which would have provided significant breadth in discussion. In fact, data saturation was reached by the last session as there were no further new emerging themes. Further research is needed to determine if health humanities interventions can further impact participants beyond the sessions, especially in their responses to future pandemics and in their mental wellness as the pandemic continues to rage on locally and throughout the world.

\section{Conclusion}

As the health humanities is gradually integrated into mainstream medical school and postgraduate medical curricula, just-in-time interventions have a role in providing healthcare professionals with education and support through the current worldwide pandemic. While the focus of this study was on the implementation of a timely film screening and discussion, the themes that emerged from the guided reflections were insightful and can inform future pandemic-preparedness efforts for frontline healthcare staff.

\section{References}

Aldrees, Turki, Mohammed Al Ghobain, Abdullah Alenezi, Saleh Alqaryan, Dana Aldabeeb, Najed Alotaibi, Kamal Alzahrani, and Sami Alharethy. 2017. "Medical Residents' Attitudes and Emotions Related to Middle East Respiratory Syndrome in Saudi Arabia.” Saudi Medical Journal 38 (9): 942-947. 
Alexander, Matthew, Mary N. Hall, and Yvonne J. Pettice. 1994. "Cinemeducation: An Innovative Approach to Teaching Psychosocial Medical Care.” Family Medicine 26 (7): 430-433.

Baños, Joseph-Eladi, and Fèlix Bosch. 2015. "Using Feature Films as a Teaching Tool in Medical Schools." Educación Médica 16 (4): 206-211.

Darbyshire, Daniel, and Paul Baker. 2012. "A Systematic Review and Thematic Analysis of Cinema in Medical Education.” Medical Humanities 38:28-33.

Ginsburg, Shiphra, and Lorelei Lingard. 2006. "Using Reflection and Rhetoric to Understand Professional Behaviours." In Measuring Medical Professionalism, edited by David Thomas Stern, 195-212. New York: Oxford University Press.

Kam, Kai-Qian, Chee Fu Yung, Lin Cui, Raymond Tzer Pin Lin, Tze Minn Mak, Matthias Maiwald, Jiahui Li, Chia Yin Chong, Karen Nadua, Natalie Woon Hui Tan, and Koh Cheng Thoon. 2020. "A Well Infant with Coronavirus Disease 2019 with High Viral Load." Clinical Infectious Diseases. https://doi.org/10.1093 /cid/ciaa201.

Kolb, David Allen. 2014. Experiential Learning: Experience as the Source of Learning and Development. New Jersey: Pearson Education, Inc.

Jianbo Lai, Simeng Ma, Ying Wang, Zhongxiang Cai, Jianbo Hu, Ning Wei, Jiang Wu, Hui Du, Tingting Chen, Ruiting Li, Huawei Tan, Lijun Kang, Lihua Yao, Manli Huang, Huafen Wang, Gaohua Wang, Zhongchun Liu, and Shaohua Hu. 2019. "Factors Associated with Mental Health Outcomes among Health Care Workers Exposed to Coronavirus Disease 2019.” JAMA Network Open 3 (3): e203976.

Powley, Elaine, and Roger Higson. 2005. The Arts in Medical Education: A Practical Guide. Oxford: CRC Press.

Rambaldini, Gloria, Kumanan Wilson, Darlyne Rath, Yulia Lin, Wayne L. Gold, Moira K. Kapral, and Sharon E. Straus. 2005. "The Impact of Severe Acute Respiratory Syndrome on Medical House Staff: A Qualitative Study." Journal of General Internal Medicine 20 (5): 381-385.

Sandars, John. 2009. "The Use of Reflection in Medical Education: AMEE Guide No. 44.” Medical Teacher 31 (8): 685-695.

Self, Donnie J. 1993. "The Educational Philosophies behind the Medical Humanities Programs in the United States: An Empirical Assessment of Three Different Approaches to Humanistic Medical Education." Theoretical Medicine 14 (3): 221-229.

Tan, Benjamin Y. Q., Nicholas Y. S. Chew, Grace K. H. Lee, Mingxue Jing, Yihui Goh, Leonard L. L. Yeo, Ka Zhang, Howe-Keat Chin, Aftab Ahmad, Faheem Ahmed Khan, Ganesh Napolean Shanmugam, Bernard P. L. Chan, Sibi Sunny, Bharatendu Chandra, Jonathan J. Y. Ong, Prakash R. Paliwal, Lily Y. H. Wong, Renarebecca Sagayanathan, Jin Tao Chen, Alison Ying Ying Ng, Hock Luen Teoh, Cyrus S. Ho, Roger C. Ho, and Vijay K. Sharma. 2020. "Psychological Impact of the COVID-19 Pandemic on Health Care Workers in Singapore.” Annals of Internal Medicine. https://doi.org/10.7326/M20-1083.

Tan, Chorh-Chuan. 2006. "SARS in Singapore - Key Lessons from an Epidemic." Annals of the Academy of Medicine Singapore 35 (5): 345-349.

Vygotsky, Lev. 1978. Mind in Society. London: Harvard University Press.

Publisher's Note Springer Nature remains neutral with regard to jurisdictional claims in published maps and institutional affiliations. 